\title{
Variations
}

Variations

Revue internationale de théorie critique

$8 \mid 2006$

Subjectivités libres et critique de la répression

\section{Walter Benjamin, ou la possibilité de ne pas trahir l'enfance}

Soizic Bonvarlet

\section{(2) OpenEdition}

Journals

Édition électronique

URL : http://journals.openedition.org/variations/503

DOI : 10.4000/variations.503

ISSN : 1968-3960

Éditeur

Les amis de Variations

Édition imprimée

Date de publication : 1 novembre 2006

Pagination : 50-58

Référence électronique

Soizic Bonvarlet, «Walter Benjamin, ou la possibilité de ne pas trahir l'enfance », Variations [En ligne], 8 | 2006, mis en ligne le 27 décembre 2012, consulté le 02 mai 2019. URL : http://

journals.openedition.org/variations/503 ; DOI : 10.4000/variations.503

Ce document a été généré automatiquement le 2 mai 2019.

Les ami•e•s de Variations 


\title{
Walter Benjamin, ou la possibilité de ne pas trahir l'enfance
}

\author{
Soizic Bonvarlet
}

1 Pour l'inclassable Walter Benjamin, enfance et révolution n'ont jamais été bien éloignées. Le critique allemand, plus utopiste que nostalgique, a ainsi rassemblé un recueil de ses propres souvenirs sous le titre d'Enfance berlinoise. Les trente textes ont été écrits entre 1932 et 1933, sous le signe du suicide et de l'arrivée au pouvoir du nazisme. En effet, en 1932, à l'âge de quarante ans, Benjamin est tenté de mettre fin à ses jours ; la raison pour laquelle il décide finalement de ne pas aller jusqu'au bout de son acte reste mystérieuse. En mars 1933, suite à la prise de pouvoir nazie, il prend la route de l'exil. En février, il avait pris soin d'envoyer à son ami Gershom Scholem, à Jérusalem, le manuscrit achevé d' Enfance berlinoise, en un geste symbolique par lequel l'enfance, au moins, était mise à l'abri des feux nazis. La publication de l'œuvre probablement la plus personnelle de Benjamin eut lieu juste dix ans après son suicide à la frontière franco-espagnole, épuisé et désespéré par la tournure funeste que prenait sa fuite pour tenter d'échapper aux nazis. Cette publication, en 1950, fut l'œuvre de Theodor W. Adorno. À travers ces récits sur l'enfance émane un profond désir de résister à la barbarie, et ce par l'affirmation de l'expérience individuelle.

2 En effet, contrairement aux apparences, l'enfant vit dans l'expérience pleinement autonome. Il est à lui tout seul flâneur, collectionneur, joueur et insurgé ; autrement dit, il est l'individu pluriel, regroupant toutes les effigies de prédilection de Benjamin. L'enfant n'est pas marqué par l'habitude, qui crée de la résignation, ni par les critères de l'utile ou de l'impossible, ennemis de l'utopie. Cela en fait un être résolument libre, malgré les tentatives de domination de son entourage, en l'occurrence celle que le père exerçait sur le jeune Benjamin.

3 Néanmoins, peut-être la mise en garde essentielle de Benjamin est-elle la suivante : ne pas bafouer l'enfant le plus proche de nous, à savoir celui qui survit à travers les adultes que nous sommes tous devenus. Car cet enfant nous chuchote des promesses de liberté et de bonheur, il est l'utopiste qui en nous ne demande qu'à se lever. 


\section{Un enfant berlinois révolté : éléments autobiographiques}

Dans les notes préliminaires à Enfance berlinoise, Benjamin se définit comme « un enfant de bonne bourgeoisie ${ }^{1} »$. Le thème essentiel du recueil est effectivement la position de l'enfant en tant qu'individu dans le milieu de la grande bourgeoisie au tournant du siècle, ainsi que ses errances dans la grande ville moderne, qui constituent tant un moyen de faire l'apprentissage du monde et de soi-même que d'échapper à sa propre classe sociale.

Dès la prime enfance, Benjamin est effectivement en position de révolte. Contre l'ordre et le conformisme de l'appartement bourgeois d'abord, tout entier dédié à la présence paternelle, c'est-à-dire à la domination. C'est ainsi que le labyrinthe ultramoderne et chaotique qu'est le Berlin du début du XXe siècle représente pour le jeune Benjamin un lieu incomparable d'émancipation, par le jeu et l'émerveillement. La ville contraste avec l'appartement, sordide refuge de la "société », que l'enfant associe à une sorte de monstre et dont il est exclu bien qu'il soit conscient qu'il s'agisse de son "clan». Benjamin écrit à propos de ce monstre inquiétant : «Et comme l'abîme qui l'avait craché était celui de ma propre classe, je faisais ainsi, ces soirs-là, pour la première fois, connaissance avec elle. Elle ne m'inspirait pas confiance ${ }^{2}$.» L'esprit critique est déjà très développé, bien que rentré, l'enfant n'ayant pas encore les moyens d'exprimer sa révolte. Il est fort possible que Benjamin, enfant mais surtout adolescent, ait ressenti une frustration douloureuse, notamment due à l'incapacité physique de résister à son propre père, et à l'impossibilité de subvenir à ses propres besoins s'il avait décidé de fuir la prison de sa classe.

La critique de l'intérieur bourgeois est donc récurrente, le bien-être et le confort n'étant qu'illusoires. En effet, «la misère ne pouvait avoir aucune place dans ces pièces où la mort ellemême n'en avait pas ». Il est vrai que les habitants de ces luxueuses demeures mouraient le plus souvent dans des sanatoriums et autres hôpitaux, jamais dans leurs appartements, "c'est pour cette raison qu'ils semblaient si confortables le jour, et devenaient la nuit le théâtre de mauvais rêves ${ }^{3}$ ". La mort, que l'on a tenté de refouler dans la recherche matérielle de sécurité, prend sa revanche nocturne dans les cauchemars. L'enfant semble être le seul à percevoir la dimension morbide du lieu dans lequel il vit.

D'ailleurs, certains objets incontournables de l'appartement bourgeois provoquent chez le jeune enfant une peur violente. C'est le cas du téléphone, dont la sonnerie troublait la sieste du père, rare moment de répit pour l'enfant, qui à cet instant était en proie à une "terreur ${ }^{4}$ » irrépressible. Ainsi l'agression sonore révélait un agresseur bien plus redoutable en la personne du père. Comme Franz Kafka dont Benjamin s'est toujours senti très proche en esprit, ce dernier eut des rapports très tendus avec la figure paternelle. La souffrance de l'enfant quant aux excès d'appétit de domination de son père est à l'origine des relations fort conflictuelles qu'il eut plus tard avec lui, et peut-être cela a-t-il contribué à renforcer son refus inflexible des formes bourgeoises d'existence.

8 L'école n'était pas un lieu préférable à l'appartement, elle n'était en aucun cas une échappatoire à la domination. Dans le texte intitulé «La Fièvre ", Benjamin écrit à propos des heures de classe manquées lorsqu'il était souffrant: "Elles ne m'apparaissaient aucunement grises et monotones comme celles que j'avais suivies. ${ }^{5}$ » Il évoque également «la misère de la stérile activité scolaire ${ }^{6} »$. Le seul moment que l'enfant appréciait était celui qui était consacré à la lecture, qui lui permettait d'échapper aux situations de 
domination. La bibliothèque des élèves était un objet de fascination. Quant aux autres objets de la salle de classe, pupitres, dictionnaires et compas, l'auteur va jusqu'à les qualifier d'«instruments de supplice ${ }^{7}$ ». Il est certain que les méthodes d'éducation scolaire en Allemagne au tournant du XXe siècle étaient marquées par la force, par une autorité démesurée des professeurs qui faisait souvent écho à celle des pères. Au lycée Wilhelmien, l'enfermement, la promiscuité, les coups, les retenues et autres humiliations arbitraires ont profondément marqué le jeune Benjamin.

Outre le sentiment de révolte qu'il nourrissait contre son père, l'école ou encore contre l'ordre inéluctable de l'appartement bourgeois, le jeune Benjamin se sentait prisonnier des quartiers riches de la capitale allemande, et ressentait un profond besoin de s'évader. Il abhorrait les quartiers du vieil et du nouvel ouest de Berlin : «Mon clan habitait alors ces deux quartiers avec une attitude où se mêlaient opiniâtreté et fierté, et qui faisaient d'eux un ghetto qu'il considérait comme son fief" ». Le jeune enfant méprisait déjà "ce quartier de possédants », et s'y sentait probablement étranger.

Mais plus généralement, Berlin fut pour lui une extraordinaire énigme à déchiffrer, l'endroit idéal à explorer en s'y égarant. Si, pour l'adulte, la grande ville moderne fut représentée par les découvertes successives de Paris, Marseille, Moscou ou encore Naples, pour l'enfant l'image du labyrinthe citadin correspond exclusivement à Berlin, haut lieu de modernité en 1900. À l'expansion industrielle dont le rythme s'accélère progressivement dans le dernier tiers du XIXe siècle correspond celle de l'urbanisme. Berlin, résidence de l'empereur d'Allemagne depuis 1870, finit par s'identifier avec le "Grand Berlin », et en quelques décennies multiplie de deux à trois fois sa population et sa superficie ${ }^{9}$. Ville d'immigration, il y a alors Berlin la même agitation que dans les métropoles nord-américaines, et le même type d'architecture résolument moderne. Benjamin évoque souvent les constructions métalliques et en verre, les réclames et les grands magasins. La publicité est déjà omniprésente. L'enfant est à la fois effrayé et fasciné par l'ampleur et l'agitation de la ville. Même les parcs participent de cette modernité urbaine. Le Tiergarten, Bois de Boulogne de Berlin, est l'un des lieux essentiels de la géographie enfantine de Benjamin. Ce qui l'attire, c'est tout ce que la grande ville recèle de labyrinthes et de dédales : s'y perdre est un art, et "s'égarer dans une ville comme on s'égare dans une forêt demande toute une éducation ${ }^{10}$ ». Le principal apprentissage de Benjamin durant son enfance est l'éducation à la ville, qui recèle d'innombrables expériences. C'est en s'y égarant et en s'éloignant du «fief » familial qu'il découvre la pauvreté, les inégalités, la prostitution, mais aussi la guerre, et ce par l'entremise de la colonne de la victoire près du Reichstag, commémorant la bataille de Sedan du 1er septembre 1870. Benjamin écrit avoir été intéressé par cette guerre qui en même temps créait chez lui un sentiment de malaise ${ }^{11}$. Déjà percevait-il probablement l'injustice infligée aux vaincus et oubliés de l'histoire, thème essentiel de sa pensée historique. Enfance berlinoise témoigne de cette perspicacité des enfants, qui pressentent nombre d'injustices et de situations de domination, perspicacité qui souvent est refoulée par l'adulte au profit d'un aveuglement volontaire servant le conformisme d'un monde « raisonnable » et ordonné. En d'autres termes, l'enfant dispose d'un capital critique intact, et ce malgré, ou peut-être justement en raison de son inexpérience. Enfin, lorsqu'il ne lisait pas ou ne pouvait se perdre dans le dédale berlinois, le jeune Benjamin échappait à l'autorité à l'instant même où il devenait collectionneur. Sa première collection fut constituée de papillons. Grâce à elle, l'enfant prit contact avec la nature et la déchiffra. Il $\mathrm{y}$ avait aussi les cartes postales. Benjamin écrit que collectionner lui permettait de 
« renouveler l'ancien ${ }^{12}$ » en le faisant sien. À l'âge adulte le désir de collection, passion des enfants pour lesquels l'utilité n'a pas de sens, ou en tout cas pas le même sens, et pour qui les choses n'ont pas encore valeur de marchandises, persistera. Il s'agira notamment de jouets en bois; collectionneur et joueur ne faisant donc qu'un. Le flâneur vient s'y ajouter, car «à votre avis, où en Allemagne pourrait-on faire cette promenade au royaume des jouets, si ce n'est dans un grand magasin berlinois ${ }^{13}$ ?» Or, les grands magasins et autres galeries commerciales, autrement dit les "passages", sont les lieux de prédilection du flâneur.

11 En outre, le jouet est l'objet inutile par excellence, et ce "par la grâce de la miniaturisation ${ }^{14} »$, comme l'écrit Giorgio Agamben, puisque des objets aussi utilitaires qu'une automobile ou qu'une cuisinière sont débarrassés de leur dimension fonctionnelle concrète, de leur "teneur chosale ", eût dit Benjamin, bien que le rite d'usage soit conservé. «Le pays des jouets, écrit Agamben, est un pays dont les habitants célèbrent des rites, ou manipulent des objets et des formules sacrées, dont ils ont pourtant oublié le sens et la fonction».

12 La collection est un réflexe enfantin, et il n'est donc pas étonnant que les objets rassemblés soient liés à cet âge. Chez Benjamin, il s'agit non seulement d'objets mais aussi de fragments de l'histoire enfantine: Enfance berlinoise est une de ses nombreuses collections, à la fois la plus personnelle et la plus universelle. Les émissions radiophoniques destinées à la jeunesse, réalisées par Benjamin entre 1929 et 1932, réunies dans le volume intitulé Lumières pour enfants, peuvent se lire dans la continuité d'Enfance berlinoise. Ces récits hantés par le merveilleux sont inspirés des contes que Benjamin parcourait lorsqu'il était très jeune, mais aussi de rêves et cauchemars enfantins, et se déroulent essentiellement dans le grand labyrinthe qu'est Berlin. Ils prouvent une nouvelle fois que Benjamin était avant tout un collectionneur d'histoires.

\section{Enfance, poésie et révolution}

13 La manière dont les enfants appréhendent les choses n'est pas régie par l'utilité mais plutôt par l'énigme, le mystère. En outre, faire que le quotidien apparaisse énigmatique définit le surréalisme dont Benjamin se considère comme entièrement redevable pour son propre travail. Les choses les plus banales prennent à travers le prisme du regard enfantin une dimension fantastique, mystérieuse. Ainsi, le jardin public devient forêt, la gare ou le passage une grotte, la ville est labyrinthe, monde fantastique. Or le labyrinthe est dénué de point d'arrivée, il s'oppose au chemin, qui représente un système fait d'opportunités, un moyen d'arriver à un but. L'enfant, lui, ne comprend pas ce qu'est l'utile le but, il n'a pas d'objectif prédéfini, pas d'arrière-pensée lorsqu'il s'intéresse à une chose, et peut ainsi se placer au-delà de la cohérence et de la rationalité instrumentale. Il s'intéresse à la chose pour elle-même, ne cherche pas à l'exploiter pour servir des intérêts personnels.

14 Le labyrinthe est par excellence le lieu où se perdre, et l'égarement est causé par le détour, la flânerie. Le flâneur, le collectionneur et le joueur sont pour Benjamin des incarnations diverses de l'oisiveté, qui représente la vraie manière de contempler les choses. Or, l'enfant est la figure qui regroupe tous ces types d'existence, car l'enfant est flâneur, collectionneur et joueur, Benjamin le montre bien assez dans les souvenirs d' Enfance berlinoise. Or, il apparaît que ces souvenirs de collections, de jeux et de flâneries sont une réalité générale de l'enfance, non particulière à Benjamin. Pour lui, la 
confirmation réside dans le fait que lorsqu'il fit lire le manuscrit de souvenirs berlinois à son ami Gershom Scholem, ce dernier eut le sentiment de se rappeler sa propre enfance.

La figure du flâneur nous conduit à évoquer Baudelaire, qui définissait ce type d'oisif comme aimant la solitude, mais dans la foule. Comme l'enfant, le flâneur est à la fois fasciné et terrifié par la foule dans laquelle il se noie. Il se laisse emporter, ce qui procure une sensation grisante et vertigineuse. Baudelaire a écrit: "L'enfant voit tout en nouveauté ; il est toujours ivre». On pourrait parler, pour l'enfance comme pour la flânerie, d' « ébranlement du moi par l'ivresse ${ }^{15}$ ", expression que Benjamin utilise pour caractériser le surréalisme. L'enfant est continuellement stupéfait, il ne connaît pas le dégrisement de l'habitude, du moins pas encore. Chaque chose conserve un pouvoir intact de « choc ». Selon Agamben, cela signifie en fait ne pas faire l'expérience de la chose, "parce qu'elle gît au fond de l'inconnu ${ }^{16}$ ». Ici l'expérience est synonyme de familiarisation, d'habitude qui se tisse autour de la chose et détruit sa nouveauté. Ainsi, le nouveau est en fait l'inexpérimentable, et Agamben constate que la poésie moderne depuis Baudelaire se fonde sur un manque d'expérience sans précédent: "L'étrangeté conférée aux objets les plus communs, pour les faire échapper à l'expérience, devient ainsi la caractéristique d'un projet poétique visant à faire de l'Inexpérimentable le nouveau "lieu commun", la nouvelle expérience de l'humanité». Cette phrase évoque immédiatement celle de Benjamin à propos du surréalisme: "Nous ne pénétrons le mystère que pour autant que nous le retrouvons dans le quotidien, grâce à une optique dialectique qui reconnaît le quotidien comme impénétrable et l'impénétrable comme quotidien ${ }^{17}$."

En outre, le manque d'expérience, qui préserve l'effet de choc que les choses exercent sur l'individu, préserve également l'esprit critique de l'individu, on le constate chez Baudelaire comme chez l'enfant. Le choc qui s'exerce, loin d'être une sorte de contrainte ou de pouvoir magique pesant sur l'individu et réduisant sa liberté, protège sa liberté. Ce choc, causé par le manque d'expérience, entretient l'intensité du sentiment ou de l'impression immédiate, que cette impression soit positive ou négative. Car il ne laisse pas le voile de l'habitude s'installer, cette habitude qui arrondit les angles, trouble la vue, et finit par tout faire accepter, "puisque la vie est ainsi ». Seule l'habitude nous contraint, d'ailleurs ne parlons-nous pas de «la force de l'habitude»? Au contraire, le choc maintient l'indignation, la hargne de la révolte, et préserve de la résignation, du renoncement, et donc de la soumission.

17 L'intérêt de Benjamin pour Baudelaire remonte aux années 1914-1915. Un livre portant sur le poète français est envisagé dès janvier 1924, date à laquelle Benjamin évoque dans des lettres à Florens Christian Rang et à Hugo von Hofmannsthal le «baroque de la banalité » qui régit l'oeuvre de Baudelaire. Les Fleurs du mal ont en effet été le premier recueil lyrique visant à rapprocher l'événement banal de l'événement poétique, et à avoir utilisé un vocabulaire à la fois prosaïque et urbain. C'est ainsi que Benjamin parle également de "technique du putsch", et qu'à partir de 1938, la forme du livre se précisant, la figure de Baudelaire se mêle à celle de Blanqui, «le plus important des chefs de barricade ${ }^{18}$ ». En d'autres termes, c'est l'image du flâneur qui s'unit à celle de l'insurgé. C'est ainsi que Benjamin écrit : «L'action de Blanqui a été la soeur du rêve de Baudelaire 19 ». La métaphore, qui abolit le sens traditionnel, est un geste de révolte pour Benjamin, et les images de Baudelaire, "originales par la bassesse des comparaisons ${ }^{20}$ ", prouvent que le poète n'a pas eu peur de jouer avec le langage, de conspirer avec lui.

18 Jouer et conspirer. Benjamin observe le cortège des poètes joueurs et des enfants conspirateurs. Évidemment, les surréalistes en font partie, «enfants adoptifs de la 
révolution ${ }^{21}$ ", qui accèdent à un au-delà de la cohérence en jouant avec la langue endehors du sens, en se plaçant "entre langue pure et langage humain ${ }^{22}$ ", position intermédiaire de l'enfance selon Agamben. Ils émancipent les choses et les êtres, en les libérant des catégories du sens, de l'utilité et de la continuité, en poussant la littérature «jusqu'aux limites extrêmes du possible ${ }^{23}$ ». Benjamin écrit également dans son article sur le surréalisme : «Il ne restait plus le moindre interstice pour y glisser le petit sou du sens. La préséance est donnée à l'image et au langage ${ }^{24}{ }$. Avec Baudelaire puis le lyrisme surréaliste, la poésie passe d'une attitude à une autre: de contemplative, elle devient véritablement révolutionnaire.

Baudelaire, Blanqui ou les surréalistes sont des constantes de l'univers à la fois politique, poétique et intellectuel de Benjamin, car ils n'ont pas trahi l'enfant en révolte qu'ils ont été. Car tout enfant est un révolté. À l'adulte de faire le choix d'exhumer ou non du passé les espoirs et promesses enfouis de son enfance. Selon Jean Lacoste, qui a rédigé une préface à Enfance berlinoise, cette collection de miniatures autobiographiques doit probablement son existence à l'idée suivante : nous avons envers l'enfant qui est en nous la même responsabilité qu'envers les espérances toujours en souffrance du passé. L'idée d'enfance est donc directement liée chez Benjamin à une tradition des opprimés qu'il veut réhabiliter. Une histoire matérialiste qui redonne la parole aux vaincus et autres oubliés de la mémoire officielle, de l'historicisme dominant. L'enfance brise la continuité de cette histoire progressiste, par les éclairs de souvenirs qui reviennent à nous de manière soudaine et presque involontaire. Cette saisie inopinée de l'essence d'une réalité enfouie dans l'inconscient évoque bien évidemment la Recherche du temps perdu. Chaque souvenir d'Enfance berlinoise est un fragment, il ne s'agit aucunement d'un récit autobiographique relatant une histoire continue. Chaque fragment contient la promesse utopique d'un monde où les choses, la nature et les hommes seraient définitivement libérés de la nécessité d'apparaître utiles, et donc de l'exploitation. Les hommes, en l'occurrence, cesseraient de subir la domination, mais aussi de l'exercer. Ainsi, un avenir utopique est secrètement enfoui dans le passé, dans les rêves de l'enfance qui doivent être respectés et considérés avec sérieux par l'adulte.

\section{L'enfant, un utopiste debout}

Benjamin esquisse un rapport à la nature totalement nouveau: celle-ci n'a pas à être domptée pas plus qu'à être considérée comme un ensemble de matières brutes utiles à la bonne vie de l'homme et donc entièrement à sa disposition. Elle n'a pas pour vocation la soumission à des besoins humains illimités, mais au contraire, elle est actrice dans un «jeu harmonien » avec l'individu, car «dans le jeu s'ébauche la première forme d'expérience individuelle ${ }^{25}$ ». L'asservissement de la nature va de pair avec la destruction de l'individu. En dévastant la nature, l'homme se consume lui-même, s'auto-détruit sans même en avoir conscience. Une fois encore, le jeu est émancipateur, il libère homme et nature de l'asservissement. Ce jeu est la clef de la "seconde technique», qui consacre le temps de l'harmonie entre la nature et l'individu, et la fin d'une vision de la nature qui serait à la merci du groupe et entièrement exploitable, ce qui résume l'état d'esprit de la première technique.

21 Ainsi donc, la révolution et le jeu, qui ont lieu dans l'ivresse, sont un même processus, ils mettent la seconde technique sur le devant de la scène et c'est ainsi que les forces productives sont libérées. L'enfant, seigneur du jeu, est par là même un révolutionnaire. 
Pour Benjamin, les révolutions sont «les tentatives d'innervation de la collectivité qui pour la première fois trouve ses organes dans la seconde technique ${ }^{26} »$. Cette innervation est donc une réappropriation du corps, et elle est comparée à l'apprentissage de l'enfant. "Et de même qu'un enfant qui apprend à saisir tend la main vers la lune comme vers une balle à sa portée - l'humanité, dans ses tentatives d'innervation, envisage, à côté des buts accessibles, d'autres qui ne sont d'abord qu'utopiques ${ }^{27} »$. L'enfant repousse sans cesse les limites du possible, c'est en ceci qu'il est un modèle pour l'humanité. Mais d'abord cette dernière doit substituer la seconde technique à la première, afin de libérer l'individu particulier qui ainsi reconquiert la possibilité d'une expérience autonome. À propos de la seconde technique et du nouvel «espace de jeu» qu'elle inaugure pour l'individu, Miguel Abensour écrit : "En même temps que l'individu mesure l'asservissement qu'exerçait sur lui la première technique, il s'élance "sans mesure" vers cette nouvelle liberté et, tel l'enfant, il ne sait pas fixer de limite à cette innervation créatrice 28 ». L'enfant ne comprend ni les valeurs de l'utile, ni celles de l'impossible. C'est pourquoi il est d'abord une source d'inspiration pour éradiquer la première technique, entièrement basée sur ce concept aliénant d'une utilité qu'il faut à tout prix exploiter, dans la nature et chez l'homme. Mais l'enfant est aussi un faiseur d'utopie, en ce qu'il ignore les limites posées par l'impossible. Il tend sa main toujours plus haut et loin, ne craint pas de paraître insensé ; l'impossible est largement à sa portée. Il ne se laisse pas ronger par la résignation, et toujours se lance dans un nouvel exploit, qui pour lui n'en est pas un. Il refuse de se compromettre, ses désirs s'énoncent avec une spontanéité qui exige tout, tout de suite, et qui ne se laisse pas "raisonner " parce que telle exigence ou tel acte est soi-disant absurde, ou encore irréalisable selon les dires et « l'expérience » des adultes. L'enfant est un héros moderne, un utopiste acharné qui ne se laisse pas dompter par les subterfuges de l'utile et du sensé. Il n'est pas le naïf que l'on croit, il reconnait et ressent profondément les situations de domination, son sens critique n'est entamé ni par l'opportunisme ni par la complaisance et sa sensibilité est à vif. Il ne cesse jamais d'être ivre, assume sa soif de justice, d'absolu, il est Antigone, Anne Frank ou encore le " petit bossu » de la légende populaire, et que l'on peut identifier au jeune Benjamin, puisqu'il le rencontra dans un poème pour enfants, et ne cessa jamais d'y penser, tant sa propre vie fut marquée par la malchance ${ }^{29}$. Cependant, dans la maladresse du geste enfantin se loge une incomparable clarté de vue, une décision résolue qui contraste avec l'inconstance et la peur « d'oser », caractéristique des adultes. L'enfant est emporté par son ivresse, nécessaire à tout acte révolutionnaire, elle lui permet de ne pas hésiter, de ne pas reculer. L'enfant ose, agit et saisit ce qui n'était pourtant pas à portée de main, et c'est la révolte qui lui donne l'élan nécessaire.

\section{NOTES}

1. Walter Benjamin, Enfance berlinoise, suivi de Sens unique, 10-18, Collection Domaine étranger, 2000, p. 54.

2. Ibid., p. 52.

3. Ibid., p. 43.

4. Ibid., p. 22. 
5. Ibid., p. 63.

6. Ibid., p. 69.

7. Ibid., p. 75.

8. Ibid., p. 82

9. Wolfgang Ribbe, Geschichte Berlins, München, 1987, vol II, p. 697. Berlin passe de 825000 habitants en 1871, à 1677000 en 1895, et à 2082000 en 1913, avec une augmentation de $252 \%$. L'augmentation la plus impressionnante se situe dans les faubourgs.

10. Walter Benjamin, Enfance berlinoise, op. cit., p. 13.

11. Ibid., p. 20.

12. Ibid., p. 80.

13. Walter Benjamin, Lumières pour enfants, Christian Bourgois, Collection « Détroits ", 1988, p. 68.

14. Giorgio Agamben, Enfance et histoire. Destruction de l'expérience et origine de l'histoire, Payot, Petite Bibliothèque Payot/Critique de la politique, 2000, p. 89.

15. Walter Benjamin, Le Surréalisme. Le dernier instantané de l'intelligentsia européenne, in OEuvres II, Gallimard, Collection Folio/Essais, Paris, 2000, p. 116.

16. Giorgio Agamben, Enfance et histoire, op. cit., p. 54.

17. Walter Benjamin, Le Surréalisme, op. cit., p. 131.

18. Walter Benjamin, Charles Baudelaire. Un poète lyrique à l'apogée du capitalisme, Payot, Collection Critique de la politique, Paris, 1990, p. 29.

19. Ibid., p. 145.

20. Ibid., p. 142.

21. Walter Benjamin, Le Surréalisme, op. cit., p. 117.

22. Giorgio Agamben, Enfance et histoire, op. cit., p. 79.

23. Walter Benjamin, Le Surréalisme, op. cit., p. 114.

24. Ibid., p. 115.

25. Walter Benjamin, Écrits français, Gallimard, Paris, 1991, p. 182.

26. Ibid., p. 149.

27. Ibid., p. 149.

28. Miguel Abensour, L'utopie de Thomas More à Walter Benjamin, Sens et Tonka, Paris, 2005, p. 173.

29. À cet égard, se référer à l'essai de Hannah Arendt sur Benjamin, in Vies politiques, Gallimard, 1974, pp. 250-251.

\section{AUTEUR}

\section{SOIZIC BONVARLET}

Enseignante en Histoire, poursuit des études de philosophie à l'université Paris 1 